Supplement of SOIL, 3, 17-30, 2017

http://www.soil-journal.net/3/17/2017/

doi:10.5194/soil-3-17-2017-supplement

(C) Author(s) 2017. CC Attribution 3.0 License.

(c) (i)

Supplement of

\title{
Timescales of carbon turnover in soils with mixed crystalline mineralogies
}

Lesego Khomo et al.

Correspondence to: Susan Trumbore (trumbore@bgc-jena.mpg.de)

The copyright of individual parts of the supplement might differ from the CC-BY 3.0 licence. 
Figure S1. Weight percent clay times the Fe oxides measured by XRD in the ClayXRD fraction versus the Fe oxyhydroxides estimated from bulk extracts (dithionite citrate minus oxalate).

Both are expressed in weight percent of Fe ( $\mathrm{g}$ Fe/100 $\mathrm{g}$ soil) The fitted line has slope $0.4 \pm 0.1$ (95\% confidence interval), and p-value: 0.0025 . Differences between the two measurements can stem from either (1) Fe oxyhydroxide coatings on the fine earth fraction including particles $>2$ um (i.e. when $\mathrm{Fe}(\mathrm{d})-\mathrm{Fe}(\mathrm{o})$ is greater than the XRD measured Fe scaled to bulk soil), or (2) some crystalline iron phases can be extracted by oxalate as well as DCB (in which case $\mathrm{Fe}(\mathrm{d})$ $\mathrm{Fe}(\mathrm{o})$ will be less than the scaled XRD-measured Fe.

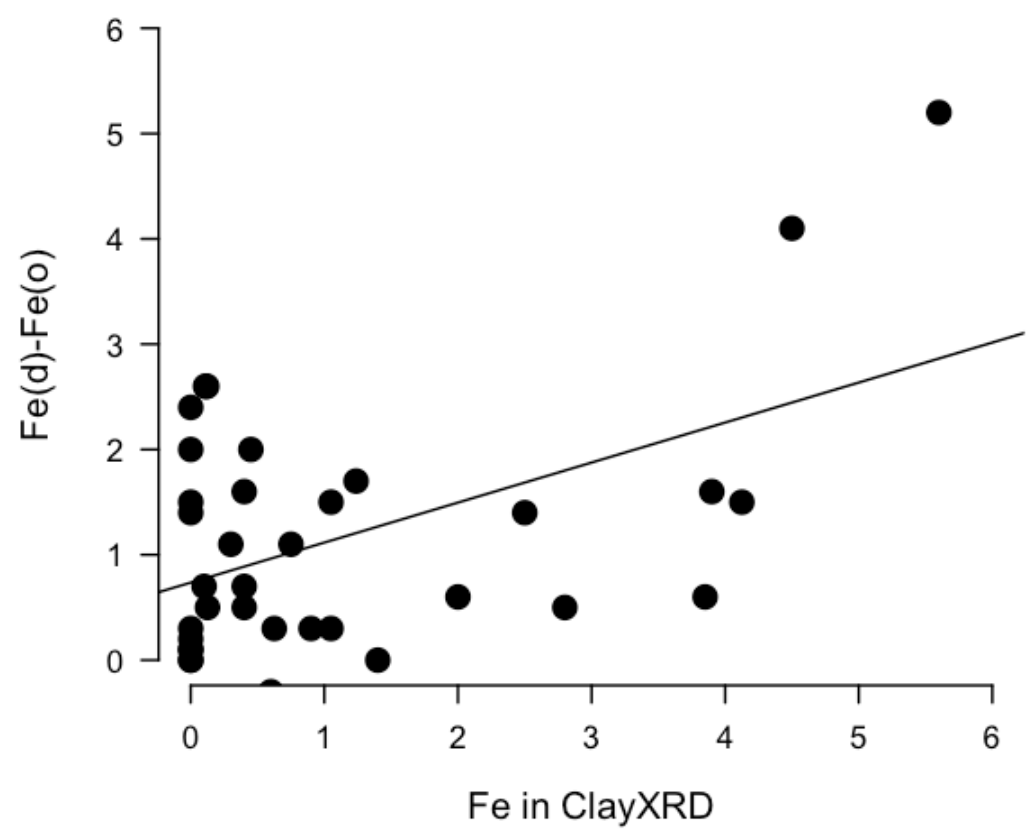

Table S1 (below) includes data used for this paper as comma-delimited text. It is also available as an excel file on request from the corresponding author.

This table contains all data from the soil profiles measured in this project.

The data are organized so that each row indicates all analyses for a given depth interval from a soil profile.

The columns are organized as follows:

\begin{tabular}{|r|l|l|}
\hline $\begin{array}{r}\text { Column } \\
\text { Number }\end{array}$ & Column Heading & Description of the Column heading \\
\hline 1 & Identifier & $\begin{array}{l}\text { For each profile, a number and letter combination } \\
\text { summarizing parent material lithology and annual } \\
\text { rainfall }\end{array}$ \\
\hline 2 & LAB ID & Identification number for Chadwick laboratory \\
\hline 3 & PIT & Pit name (field notes) \\
\hline
\end{tabular}




\begin{tabular}{|c|c|c|}
\hline 4 & geology & lithology of the parent rock \\
\hline 5 & rainfall & $\begin{array}{l}\text { mean annual precipitation in } \mathrm{mm} \text { per year at sampling } \\
\text { site }\end{array}$ \\
\hline 6 & DATE & $\begin{array}{l}\text { Year of soil sampling (important for radiocarbon } \\
\text { modeling) }\end{array}$ \\
\hline 7 & EASTING & Latitude of soil pit \\
\hline 8 & NORTHING & Longitude of soil pit \\
\hline 9 & DEPTH $1(\mathrm{~cm})$ & Top depth of horizon sampled $(\mathrm{cm})$ \\
\hline 10 & DEPTH $2(\mathrm{~cm})$ & Bottom depth of horizon sampled $(\mathrm{cm})$ \\
\hline 11 & Midpoint $(\mathrm{cm})$ & midpoint depth of horizon sampled $(\mathrm{cm})$ \\
\hline 12 & THICKNESS $(\mathrm{cm})$ & thickness of horizon $(\mathrm{cm})$ \\
\hline 13 & COLOR & Munsell color (at field moisture) \\
\hline 14 & TEXTURE & standard classification; sl = silt loam \\
\hline 15 & STRUCTURE & standard classification \\
\hline 16 & HORIZON & standard classification \\
\hline 18 & ROOTS & standard classification \\
\hline 19 & GRAVEL (\%) & $\begin{array}{l}\text { per cent of total soil volume estimated to consist of } \\
\text { gravel sized rock }\end{array}$ \\
\hline 20 & Fraction fines & $\begin{array}{l}\text { Fraction fines: fraction of total soil volume estimated to } \\
\text { be less than gravel sized }\end{array}$ \\
\hline 21 & mass fines & $\begin{array}{l}\text { mass fines: fraction of fines * bulk density }(\mathrm{g} \mathrm{cm}-3) * \\
\text { horizon thickness }(\mathrm{cm}) * 10^{\wedge} 4 \mathrm{~cm}^{\wedge} 2^{*} \mathrm{~m}^{\wedge}-2^{*} 1000 \mathrm{~kg} / \mathrm{g} \text {, } \\
\text { final units are } \mathrm{kg} \mathrm{m} \mathrm{m}^{\wedge}-2\end{array}$ \\
\hline 22 & est. BD & $\begin{array}{l}\text { bulk density }(\mathrm{g} \mathrm{cm}-3) \text { estimated using paraffin-clod } \\
\text { method. }\end{array}$ \\
\hline 23 & $\mathrm{Fe}(\mathrm{d})$ & $\begin{array}{l}\text { Fe in dithionite citrate bicarbonate (DCB) extract), } \\
\text { expressed as \% (g Fe per } 100 \text { gram dry soil }(<2 \mathrm{~mm}) \\
\text { extracted) }\end{array}$ \\
\hline 24 & $\mathrm{Fe}(0)$ & $\begin{array}{l}\mathrm{Fe}(\mathrm{o}) \text { : Fe in acid ammonium oxalate (AAO) extract, } \\
\text { expressed as \% (g Fe per } 100 \text { gram dry soil }(<2 \mathrm{~mm}) \\
\text { extracted) }\end{array}$ \\
\hline 25 & $\mathrm{Fe}(\mathrm{d})-\mathrm{Fe}(\mathrm{o})$ & units are $\mathrm{g}$ Fe per $100 \mathrm{~g}$ dry weight soil \\
\hline 26 & $\mathrm{Al}(0)$ & $\begin{array}{l}\text { Al in acid ammonium oxalate (AAO) extract (expressed } \\
\text { as \% (g Al per } 100 \text { gram dry soil }(<2 \mathrm{~mm}) \text { extracted) }\end{array}$ \\
\hline 27 & Conductivity & units are micros $/ \mathrm{cm}$ \\
\hline 28 & $\mathrm{pH}$ & no units \\
\hline 29 & CEC & $\begin{array}{l}\text { Cation Exchange capacity (milli-equivalents of charge per } \\
\text { gram dry soil), determined by extracting the ammonium } \\
\text { saturated samples with a } 1 \mathrm{M} \text { potassium chloride } \\
\text { solution in a Lachat analyzer }\end{array}$ \\
\hline
\end{tabular}




\begin{tabular}{|c|c|c|}
\hline 30 & carbon-less CEC & $\begin{array}{l}\text { CEC corrected for the contribution of organic matter by } \\
\text { assuming a contribution of } 200 \mathrm{cmol}(+) \text { per kg organic C } \\
\text { (milli-equivalents of charge per gram dry soil) }\end{array}$ \\
\hline 31 & $\%$ Base Saturation & percent of CEC from base cations \\
\hline 32 & oven dry \%C & Total C, reported as \% (grams C per 100 gram soil). \\
\hline 33 & $\% \mathrm{C}$ organic & $\begin{array}{l}\text { Organic } C \text {, reported as \% (grams } C \text { per } 100 \text { gram soil). } \\
\text { These are analyzed on samples acidified to remove } \\
\text { carbonates }\end{array}$ \\
\hline 34 & LOI inorg C & $\begin{array}{l}\text { Determined as the difference between total } \mathrm{C} \text { and } \\
\text { organic C. Reported as \% (g inorganic } \mathrm{C} / 100 \mathrm{~g} \text { soil). }\end{array}$ \\
\hline 35 & oven dry \% $\mathrm{N}$ & $\begin{array}{l}\text { \%N as measured with elemental analyzer, includes } \\
\text { organic and inorganic } \mathrm{N} \text { (g N per } 100 \text { gsoil). }\end{array}$ \\
\hline 36 & $\begin{array}{l}\mathrm{kgC} \mathrm{m}-2 \text { in } \\
\text { horizon }\end{array}$ & $\begin{array}{l}\mathrm{kg} \text { of organic carbon per } \mathrm{m}^{\wedge} 2 \text { in horizon. Calculated as } \\
\text { (mass fines }(\mathrm{kg} \mathrm{m}-2) * \text { org. } \mathrm{C}(\mathrm{g} / 100 \mathrm{~g} \text { soil)*1000gsoil } / \mathrm{kg} \\
\text { soil) }\end{array}$ \\
\hline 37 & $\begin{array}{l}\mathrm{kgC} \mathrm{m}-2 / \mathrm{cm} \\
\text { depth }\end{array}$ & $\begin{array}{l}\mathrm{C} \text { density per } \mathrm{cm} \text { depth, obtained by dividing horizon } \mathrm{C} \\
\text { inventory by horizon thickness }\end{array}$ \\
\hline 38 & C:N & Organic C/LOI N \\
\hline 39 & d13CaCO3 & $\delta^{13} \mathrm{C}$ of $\mathrm{CO}_{2}$ released from acidification of soil (in \% $\mathrm{PDB}$ ) \\
\hline 40 & D14CaCO3 & $\begin{array}{l}\Delta^{14} \mathrm{C} \text { of } \mathrm{CaCO}_{3}: \Delta^{14} \mathrm{C} \text { of } \mathrm{CO}_{2} \text { released from acidification of } \\
\text { soil }(\% \text { ) } \text {, year of measurement should be assumed to be } \\
2011 \text { for conversion to fraction Modern }\end{array}$ \\
\hline 41 & d13C bulk & $\delta^{13} \mathrm{C}$ of bulk organic $\mathrm{C}$ (in \%。 PDB) \\
\hline 42 & D14C bulk & $\begin{array}{l}\Delta^{14} \mathrm{C} \text { of bulk organic } \mathrm{C}(\%) \text {, year of measurement } \\
\text { should be assumed to be } 2011 \text { for conversion to } \\
\text { fraction Modern }\end{array}$ \\
\hline 43 & bulkTT & $\begin{array}{l}\text { Turnover time (years) that yields the radiocarbon } \\
\text { signature in the year the soil; see r code in Supplemental } \\
\text { Material. }\end{array}$ \\
\hline 44 & Fraction HF in soil & grams of HF fraction per gram of bulk soil extracted \\
\hline 45 & $\% \mathrm{CHF}$ & grams $\mathrm{C}$ in $100 \mathrm{~g} \mathrm{HF}$ fraction soil \\
\hline 46 & $\mathrm{kgC} \mathrm{m}-2$ in $\mathrm{HF}$ & heavy fraction $\mathrm{C}$ density \\
\hline 47 & \%totalC in $\mathrm{HF}$ & $\begin{array}{l}\text { \%totalC in HF: calculated as ([100*fraction HF } \\
\text { (gHF/gsoil)] } \times[\% \mathrm{C} \text { in HF])/(\%C in bulk }\end{array}$ \\
\hline 48 & d13C HF & $\delta^{13} \mathrm{C}$ in heavy fraction (in \%o, PDB) \\
\hline 49 & D14C HF & $\begin{array}{l}\Delta^{14} C \text { in heavy fraction (in \%o), assume } 2011 \text { as the } \\
\text { measurement year. }\end{array}$ \\
\hline 50 & HF TT & $\begin{array}{l}\text { Turnover time (in years; determined using SoilR; Sierra } \\
\text { et al. 2014) and the Intcal } 2013 \text { southern hemisphere } \\
\text { zone } 1,2 \text { atmospheric } 14 \text { C record. }\end{array}$ \\
\hline 51 & grav fraction LF & grams of LF fraction per gram of bulk soil extracted \\
\hline 52 & $\% C$ LF & grams $C$ in $100 \mathrm{~g}$ root free free light fraction (density \\
\hline
\end{tabular}




\begin{tabular}{|c|c|c|}
\hline & & $<1.6 \mathrm{~g} \mathrm{~cm}-3$ ) soil \\
\hline 53 & $\mathrm{kgC} \mathrm{m}-2$ in LF & light fraction $\mathrm{C}$ density \\
\hline 54 & \%totalC in LF & $\begin{array}{l}\text { alculated as ([100*fraction LF (gLF/gsoil)] } \times[\% \mathrm{C} \text { in } \\
\mathrm{HF}]) /(\% \mathrm{C} \text { in bulk soil) }\end{array}$ \\
\hline 55 & $\%$ Croots & $\begin{array}{l}\text { visible roots were picked from the LF fraction, this is the } \\
\mathrm{gC} / 100 \mathrm{~g} \text { roots }\end{array}$ \\
\hline 56 & d13Croots & $\delta^{13} \mathrm{C}$ of roots picked from $\mathrm{LF}$ (in \% PDB) \\
\hline 57 & D14Croots & $\begin{array}{l}\Delta^{14} \mathrm{C} \text { of roots picked from LF (\%), year of } \\
\text { measurement should be assumed to be } 2011 \text { for } \\
\text { conversion to fraction Modern }\end{array}$ \\
\hline 58 & $\% \mathrm{CLF}$ & $\begin{array}{l}\mathrm{gC} / 100 \mathrm{~g} \text { combusted of the } \mathrm{LF} \text { fraction after removal of } \\
\text { roots }\end{array}$ \\
\hline 59 & d13CLF & $\delta^{13} \mathrm{C}$ in root-free fLF (in \%o) \\
\hline 60 & D14C LF & $\begin{array}{l}\Delta^{14} \mathrm{C} \text { in root-free LF (in \%o, using } 2010 \text { as the date } \\
\text { of measurement) }\end{array}$ \\
\hline 61 & LFTT short & $\begin{array}{l}\text { Turnover time estimated from } \Delta^{14} C \text { of the root-free fLF } \\
\text { - where two solutions are possible, the shorter of the } \\
\text { two (in years) }\end{array}$ \\
\hline 62 & LFTT long & $\begin{array}{l}\text { Turnover time estimated from } \Delta^{14} \mathrm{C} \text { of the root-free } \\
\text { fLF - where two solutions are possible, the longer of } \\
\text { the two (in years) }\end{array}$ \\
\hline 63 & $\%$ C clay & $\begin{array}{l}\mathrm{gC} \text { in } 100 \mathrm{~g} \text { of isolated clay-sized XRD fraction. This } \\
\text { fraction was also treated with } 2 \% \mathrm{H} 2 \mathrm{O} 2 \text {, so the } \mathrm{C} \text { is } \\
\text { assumed to be strongly associated with clay } \\
\text { surfaces. This is the same fraction used for } \\
\text { mineralogy analysis by XRD. }\end{array}$ \\
\hline 64 & \%total C in clay & $\begin{array}{l}\text { calculated as }\left(\left[100^{*} \text { gravimetric fraction clay }(\mathrm{g}\right.\right. \\
\text { clay/g soil })] \times[\% \mathrm{C} \text { in clay }]) /(\% \mathrm{C} \text { in bulk soil) }\end{array}$ \\
\hline 65 & d13 clay & $\delta^{13} \mathrm{C}$ of the clay-sized XRD fraction(in \%०) \\
\hline 66 & D14C clay & $\begin{array}{l}\Delta^{14} \mathrm{C} \text { of } \mathrm{C} \text { in the Clay XRD fraction, using } 2011 \text { as } \\
\text { the date of measurement) }\end{array}$ \\
\hline 67 & TT clay & $\begin{array}{l}\text { Turnover time (years) estimated from } \Delta^{14} \mathrm{C} \text { that } \\
\text { yields the radiocarbon signature in the clayXRD } \\
\text { fraction using a simple one-pool model; see r code } \\
\text { in Supplemental Material. }\end{array}$ \\
\hline 68 & $\% C$ nonclay & $\begin{array}{l}\text { (calculated using mass balance, see equations in } \\
\text { text) }\end{array}$ \\
\hline 69 & $13 \mathrm{C}$ nonclay & $\begin{array}{l}\delta^{13} \mathrm{C}-\mathrm{C} \text { nonclay-sized fraction (calculated using } \\
\text { mass balance, see equations in text) }\end{array}$ \\
\hline 70 & 14Cnonclay & $\begin{array}{l}\Delta^{14} \mathrm{C} \text { in the nonclay-sized fraction (calculated using } \\
\text { mass balance, see equations in text) }\end{array}$ \\
\hline 71 & TT nonclay & $\begin{array}{l}\text { TT of nonclay sized fraction estimated using a one- } \\
\text { box model from } \Delta^{14} \text { Cnonclay (see text) }\end{array}$ \\
\hline
\end{tabular}




\begin{tabular}{|c|l|l|}
\hline 72 & sand & Particle size \% of total mass in sand size particles \\
\hline 73 & silt & Particle size \% of total mass in silt size particles \\
\hline 74 & clay & $\begin{array}{l}\text { Particle size \% of total mass in clay size particles } \\
\text { \%clay } \% \text { of clay-sized XRD fraction that is } \\
\text { smectite/100 }\end{array}$ \\
\hline 75 & clay*smectite/100 & per cent of clay-sized XRD fraction that is Quartz \\
\hline 76 & Quartz & per cent of clay-sized XRD fraction that is Feldspars \\
\hline 77 & Feldspars & per cent of clay-sized XRD fraction that is Calcite \\
\hline 78 & Calcite & per cent of clay-sized XRD fraction that is Oxides \\
\hline 79 & Oxides & per cent of clay-sized XRD fraction that is Kaolins \\
\hline 80 & Kaolins & per cent of clay-sized XRD fraction that is Smectites \\
\hline 81 & Smectites & per cent of clay-sized XRD fraction that is Chlorites \\
\hline 82 & Chlorites & per cent of clay-sized XRD fraction that is Micas \\
\hline 83 & Micas &
\end{tabular}

Table S1. Continued. Comma-delimited text file with data (metadata given above).

$1,2,3,4,5,6,7,8,9,10,11,12,13,14,15,16,17,18,19,20,21,22,23,24,25,26,27,28$ $, 29,30,31,32,33,34,35,36,37,38,39,40,41,42,43,44,45,46,47,48,49,50,51,52,5$ $3,54,55,56,57,58,59,60,61,62,63,64,65,66,67,68,69,70,71,72,73,74,75,76,77$, $78,79,80,81,82,83,84,85,86$

Identifier, LAB ID, PIT ME, geology, rainfall, DATE, EASTING, NORTHING, DEPTH 1 (cm), DEPTH 2 ( $\mathrm{cm})$, Midpoint ( $\mathrm{cm})$, THICKNESS

(cm), COLOR, TEXTURE, STRUCTURE, HORIZON, ROOTS, GRAVEL ( $\left.\frac{\circ}{\circ}\right)$, Fraction fines, mass fines, est. $\mathrm{BD}, \mathrm{Fe}(\mathrm{d}), \mathrm{Fe}(\mathrm{o}), \mathrm{Fe}(\mathrm{d})-\mathrm{Fe}(\mathrm{o}), \mathrm{Al}(\mathrm{o})$, Conductivity

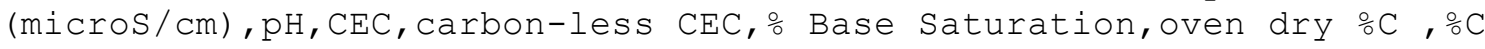

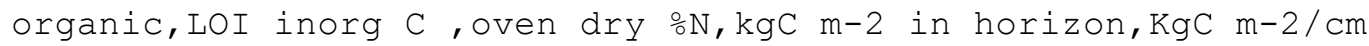
depth, C:N, d13 CO-3, D14C CO-3, d13C bulk, D14C bulk,bulkTT,Fraction HF in soil, $\frac{\mathrm{C}}{\mathrm{HF}}, \mathrm{kgC} \mathrm{m}-2$ in $\mathrm{HF}$, ototalC in $\mathrm{HF}, \mathrm{d} 13 \mathrm{C} \mathrm{HF}, \mathrm{D} 14 \mathrm{C} \mathrm{HF}, \mathrm{HF} \mathrm{TT}$, grav fraction $\mathrm{LF}, \circ \mathrm{C} \mathrm{LF}, \mathrm{kgC} \mathrm{m}-2$ in $\mathrm{LF}$, \%totalC in $\mathrm{LF}, \circ \mathrm{C}$ roots, d13C roots, $114 \mathrm{C}$ roots, $\circ \mathrm{C}$ LF, d13C LF, D14C LF, LFTT short, LFTT long, oC clay, ototal C in clay, d13 clay, D14C clay, TT clay, oC nonclay,13C nonclay,14Cnonclay, TT nonclay, Mass non-clay in horizon, cumulative mass nonclay, mass clay I nhorizon, cumulative mass

clay, sand, silt, clay, clay* smectite/100, Quartz,Feldspars, Calcite, Oxides, Kaol ins, Smectites, Chlorites, Micas

$\mathrm{RH}-450-\mathrm{C}, \mathrm{SA} 1616, \mathrm{k} 12-$

1, rhyolite, 470,2010,351375,7421676,0,3,2,3,5yr3/4, sl, 1fgr-

$\mathrm{sg}, \mathrm{A}, 1 \mathrm{vf}, 5,0.95,29,1,2.7,0.11,2.6,0.1,22,6.7,4.4,1.7,120.79,0.82,0.82,<0.0$ $1,0.08,0.23,0.08,10, \quad, \quad,-19.1,68.5,130,0.99,0.67,0.19,81.3,-$ $18.2,72.9,120,0.008,16.64,0.036,15.2, \quad, \quad, 16.64,-23.2,98.4,8.5,85,$, , $,,,, \quad, 28,28,0.3,0.3,85,17.5,1,0.6,0,1,0,12,25,57,4,1$ $\mathrm{RH}-450-\mathrm{C}, \mathrm{SA} 1617, \mathrm{k} 12-$

1, rhyolite, 470,2010,351375,7421676,3,15,9,12,5yr3/4,sl,1fgr,Bw1,1vf-fm.co.vc, 50,0.5,90,1.5,2.7,0.11,2.6,0.1,26,6.9, 10.6,8.4,32.94,0.64,0.636,<0 $.01,0.06,0.57,0.05,10, \quad, \quad,-16.4,47.5,175,0.98,0.54,0.478,83.5,-$ 
$16.2,40.9,195,0.003,1.93,0.005,0.9, \quad, \quad, \quad, 1.93,-20,76.7,5.5,112,1, \quad, \quad$, $,, \quad, 89,117,0.9,1.2,82.5,17.5,1,0.4,7,1,0,11,33,36,1,11$

$\mathrm{RH}-450-\mathrm{C}, \mathrm{SA} 1618, \mathrm{kl} 2-$

1 , rhyolite, $470,2010,351375,7421676,15,30,23,15,5 y r 3 / 4$, sl-

$\mathrm{scl} \sim 20 \%, \mathrm{fgr}, \mathrm{Bw} 2,3 \mathrm{vf}-\mathrm{f}-1 \mathrm{~m}-\mathrm{Co}-$

$\mathrm{VC}, 70,0.3,77,1.7,2.7,0.11,2.6,0.1,19,6.7,5.8,3.7,95.31,0.62,0.62,<0.01,0.0$ $7,0.48,0.03,10, \quad,-14.5,-15.4,450,, 0.62, \quad, \quad,-12.6,420,,,,,$,

$,,, 1,1,1,1,1,76,193,0.8,2,85,15,1,1,1$, 'RH-450-C', SA $1619, \mathrm{kl} 2-$

1, rhyolite, 470,2010,351375,7421676,30,60,45,30,2.5yr3/4, sl++, rock, CR, 1vf$\mathrm{Co}, 95,0.05,26,1.7,2.6,0.14,2.5,0.1,20,6.4,5.7,3.9,83.82,0.53,0.53,<0.01,0$. $06,0.14,0,9, \quad,-13.6,88.1,98,, 0.53,, \quad,-15.4,445$,

GR-450-C, SA 294,ph5c, granite, 470,2004,322713,7452153,0,23,12,23,7.5YR $2.5 / 2,1 \mathrm{~s}, \mathrm{sg}, \mathrm{BA}, 2 \mathrm{f}-\mathrm{vf}-$

$1 \mathrm{~m}, 80,0.2,74,1.6,0.6,0.05,0.5,0,0.03,6.1,10.4,7.7,72.82,0.8,0.78,<0.01,0.0$ $7,0.57,0.02,12, \quad, \quad,-20.2,30.4,210,0.97,0.47,0.335,58.7,-$

$19.5,30.1,230,37,,, \quad, \quad, 37,-23.7,99.3,5,105,3,24.1,,,, 0.63,$,

$, \quad, 69,69,4.6,4.6,77.5,16,6.25,2.9,6,4,0,2,41,46,1,0$

GR-450-C,SA 295, ph5c,granite, 470,2004,322713,7452153,23,45,34,22,7.5YR

$3 / 4, \mathrm{~s}, \mathrm{sg}, \mathrm{BC}, 1 \mathrm{f}-\mathrm{m}-$

$2 \mathrm{vf}, 90,0.1,37,1.7,0.7,0.03,0.7,0,0.01,5.9,11.6,8.9,49.1,0.8,0.36,<0.01,0.0$ $4,0.13,0.01,10, \quad,-18.4,-77.2,900,0.98,0.39,0.143, \quad,-17.5,-$

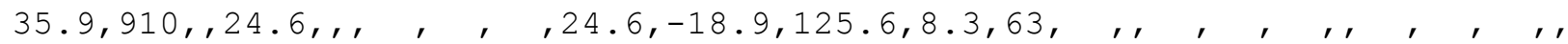
$, \quad, 82.5,13,5,2.2,6,6,0,2,41,44,2,0$

$\mathrm{NE}-450-\mathrm{C}, \mathrm{SA} 1613,1 \mathrm{e} 6-$

1, nephelinite,470,2010,336567,7398988,0,2,1,2,10yr3/2, sicl,1fgr, A, 3vf-

$\mathrm{f}, 80,0.2,4,1,4.4,0.27,4.1,0.2,113,7,55,34.4,68.68,6.04,6.04,<0.01,0.51,0.2$

$4,0.12,12, \quad, \quad,-17.8,65,135,0.92,4.18,0.154,63.7,-$

$16.7,74.4,120,0.028,31.28,0.035,14.4, \quad, \quad, \quad, 31.28,-$

$20.6,88.3,8,98,2.52,12.5,-16.9,-1.5,365,7.54,-$

$18,74.5,120,3,3,1.2,1.2,27.5,43,30,14.1,1,1,0,15,24,47,0,12$

$\mathrm{NE}-450-\mathrm{C}, \mathrm{SA} 1614,1 \mathrm{e} 6-$

1 , nephelinite, 470,2010,336567,7398988,2,18,10,16,7.5yr3/2, cl,1f-

$\operatorname{mgr}, \mathrm{Bw} 1,2 \mathrm{vf}-\mathrm{f}-$

$1 \mathrm{Vc}, 90,0.1,27,1.7,5.5,0.27,5.2,0.2,80,6.8,76.1,65.8,62.41,3.04,3.04,<0.01$, $0.26,0.83,0.05,12, \quad, \quad,-15.4,8,320,0.95,2.43,0.628,75.9,-$

$14.9,1.8,345,0.007,30.85,0.059,7.2, \quad, \quad, \quad, 30.85,-$

$19.5,64.4,4.2,133,1.14,15,-16.8,-129.5,1400,4.3,-$

$15.1,32.3,215,16,19,10.9,12.1,27.5,33,40,19.2,1,1,0,14,26,48,0,9$

$\mathrm{NE}-450-\mathrm{C}, \mathrm{SA} 1615,1 \mathrm{e} 6-$

1, nephelinite,470,2010,336567,7398988,18,40,29,22,7.5yr3/4,cl-,gr- broken rocks, BC, 1vf, 90,0.1,37,1.7,3.9,0.43,3.5,0.5,48, 7,65,58.4,58.63,1.94,1.94,< $0.01,0.19,0.73,0.03,10, \quad,-14.1,-55.2,725, \quad, \quad, \quad,-96.1,, 1,1$,

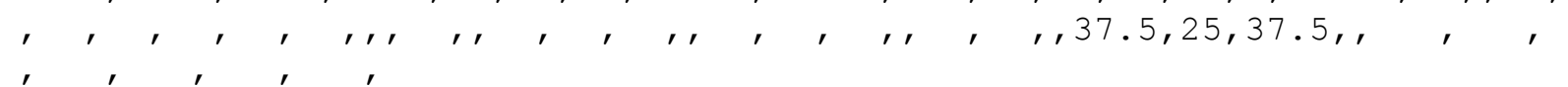

GA-450-C*, SA 1607, ph4a-

3, gabbro, 470,2010,321956,7449291,0,2,1,2,10yr2/1, sicl,pl-1f-

mgr, A, 2vf, 2, 0.98,22,1.1,1.6,0.09,1.5,0.2,131,8.1,53.4,41.8,89.1,3.4, 3.27, 0 $.13,0.25,0.7,0.35,13, \quad, \quad,-14.9,20.1,260,0.92, \quad, \quad, \quad,-$

$13.6,6.2,325,0.016,34.2,0.121,17.2, \quad, \quad, \quad, 34.2,-$ 
$16.8,44.1$, none, $185,4.69,21.6,-14.8,-64.6,800,3.01,-$

$14.9,43.4,185,18,18,3.2,3.2,52.5,32.5,15,10.1,2,2,0,7,0,67,0,22$

GA $-450-C^{*}, \mathrm{SA} 1608, \mathrm{ph} 4 \mathrm{a}-$

3, gabbro, 470,2010,321956,7449291,2,12, 7, 10,10yr3/2, cl-c, 2f-

msbk, Bw1,3vf.f.m, 3, 0.97,136,1.4,1.5,0.08,1.4,0.1,135,8.3,47.2, 35.7,133.12, $3.38,1.9,1.48,0.15,2.58,0.26,13, \quad, \quad,-13.9,-28.8,525,0.93, \quad, \quad,-$

$14.5,20.9,260,0.01,42.27,0.574,22.2, \quad, \quad, \quad, 42.27,-$

$15,34.4$, none, 215,2.35,30.8,-13.9,-145,1530,1.75,-

$13.9,23,250,102,120,34,34,47.5,27.5,25,10.8,1,9,6,10,0,43,3,28$

GA-450-C*, SA $1609, \mathrm{ph} 4 \mathrm{a}-$

3, gabbro, 470,2010,321956,7449291,12,25,19,13,10yr3/2, cl, 2f-mabk, Bw2, 2vf-

f.1Vc, 5, 0.95,185,1.5,1.4,0.08,1.3,0.1,131,8.3,45.5,33.3, 147.98, 3.57, 1.73,1

$.84,0.14,3.2,0.25,12, \quad,-14.3,-82.6,950,, 1.73, \quad, \quad,-82.6,940$, ,

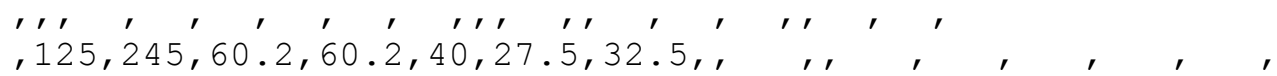

GA-450-C*, SA 1610, ph $4 \mathrm{a}-$

3, gabbro, 470,2010,321956,7449291,25,36,31,11,10yr3/3, c1,3m-

coabk, Bw3, 2vf.f.m, 10,0.9,149,1.5,1.2,0.07,1.1,0.1,134,8.4,47.1,33.5, 142.09

,4.01,1.45,2.55,0.13,2.16,0.2,11, , ,-14.8,-108.4,1200, ,1.45, , , , $108.4,780,,,,$, , , , , , , , , , , , ,

$, 93,338,55.7,55.7,37.5,25,37.5,23.6,1,1,31,2,0,63,0,1$

GA-450-C*, SA $1611, \mathrm{ph} 4 \mathrm{a}-$

3, gabbro, 470,2010,321956,7449291,36,47,42,11,10yr3/4, c,2f-msbk-sg, BC1, 1f-

$\mathrm{m}, 20,0.8,141,1.6,1.4,0.06,1.3,0.1,133,8.4,41.6,26.9,151.7,4.31,1.6,2.7,0.1$

$4,2.26,0.21,12, \quad, \quad-15,-111.1,1210, \quad, \quad, \quad, \quad,-111.1,1210,,, 1$,

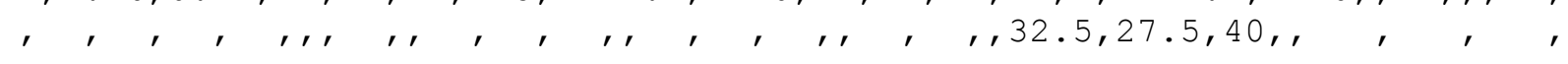

GA-450-C*, SA' 1612, ph4a-

3, gabbro, 470,2010,321956,7449291,47,70,59,23,10yr4/3, sc1, sg, BC2, 1vf, $70,0.3$ ,110,1.6,1,0.05,0.9,0.1,120,8.5,27.8,16.4,207.79,3.34,0.95,2.39,0.09,1.05, $0.05,11, \quad,-15.2,-112.3,1230, \quad, \quad, \quad, \quad,-112.3,1230$, ,

,

RB-450-C, SA 1500,le3-1, picrite/olivine-rich basalt/letaba basalt/black basalt, 470,2009,341888,7420588,0,4,2,4,10yr2/1, cl, 1vf-f-mgr, A1, 2vf-

$\mathrm{f}, 5,0.95,38,1,1.6,0.14,1.5,0.1$,

$, 7.4,51,44.4,69.92,1.94,1.94,<0.01,0.12,0.74,0.18,16, \quad,-455.3,-14.9,-$ $16,450,0.91,1.79,0.619,83.9,-14.8,-23.1,490,11.56,1,51.95,-$

$16.6,75.5,11.56,-16.6,60,2.5,145,2.5,46.6,-14.1,-125.3,1350,1.62,-$

$15.5,79.3,110,24,24,13.7,13.7,43,21,36,35.7,1,0,0,0,0,99,0,0$

RB-450-C,SA 1501, le3-1,picrite/olivine-rich basalt/letaba

basalt, 470,2009,341888,7420588,4,15,10,11,10yr2/2, cl,2f-mgr, A2, 3vf-f-

$1 \mathrm{~m}, 20,0.8,106,1.2,1.8,0.15,1.6,0.2$,

$, 7.5,46.8,40.5,86.87,1.84,1.7,0.13,0.11,1.8,0.16,16,-6.1,-697.3,-13.3,-$

$95.2,1060,0.84,1.4,1.24,68.9,-13.3,-95.2,1070,9.97,,, \quad, \quad, 9.97,-$

$16.6,30$, none, 230,2.5,55.6, , , ,1.22, , ,66,90,40,53.7,42,20,38, , ,

'RB-450-C', SA '1502,'le3-1, picrite/olivine-rich basalt/letaba

basalt, 470,2009,341888,7420588,15,30,23,15,10yr3/2, cl+,1f-m-gr, Bk1,2vf-f-

$1 \mathrm{~m}, 35,0.65,146,1.5,1.7,0.18,1.5,0.2$,

, 7.7,51.4,45.9,93.89,1.62,1.23,0.384529784,0.1,1.81,0.12,13,-2.4,-943.2,$13.3,-151.6,1490,0.83,1.38,1.676,92.8,-13.3,-151.6,1640,10,1,, \quad, 10$, $, \quad,, 2.5,85.1, \quad, \quad, 0.32, r, 85,175,61.4,38,20,42, r$, , , 
RB-450-C, SA 1503,le3-1, picrite/olivine-rich basalt/letaba

basalt, 470,2009,341888,7420588,30,49,40,19,10yr3/3, cl, m, Bk2, 1vf, 45, $0.55,15$

$7,1.5,1.5,0.13,1.4,0.2$,

$, 7.9,51.9,45.3,115.37,1.92,1.29,0.63,0.09,2.02,0.11,14,-3.7,-680,-13.3,-$

$216.2,2280,0.84,1.28,1.687,83.5,-13.3,-216.2,2410,11.18,1,33.61,-$

$12.4,76,11.18, \quad,-53$, none, $700,2.46,88.1,-13.5,-$

$192.6,2100,0.28,1,84,259,72.3,36,17,46,45.2,2,0,0,0,0,98,0,0$

RB-450-C, SA 1504,le3-1, picrite/olivine-rich basalt/letaba

basalt,470,2009,341888,7420588,49,68,59,19,10yr7/2 (calcite nodule)

$10 \mathrm{yr} 4 / 3$ (soil), cl, m,Bk3/C,1vf,90,0.1,30,1.6,1,0.12,0.9,0.2,

$, 8,46.7,32.7,129.7,4.1,0.84,3.26,0.06,0.25,0.01,14, \quad, \quad,-13.3,-$

$216.1,2280$

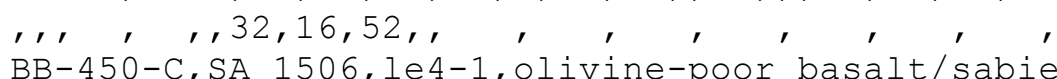

basalt, 470,2009,344120,7421754,0,3,2,3,7.5yr2.5/1, cl, 1fsbk, A1, 2vf-

$\mathrm{f}, 20,0.8,26,1.1,2.1,0.156,2,0.1$,

,6.9,41.1,32.9,85.05,2.41,2.41,<0.01,0.15,0.64,0.21,16, , ,-13.7,-

$25.4,500,0.89,1.54,0.363,57.1,-13.7,-25.4,510,10,1, \quad, \quad, 10,-$

$15.4,33.5$, none, $217,2.48,40.4,-14.3,-91.7,1030,2.36,-$

$13.4,19.5,265,16,16,10.4,10.4,38,23,39,36.1,1,0,0,0,6,92,0,0$

BB-450-C,SA 1507,le4-1, olivine-poor basalt/sabie

basalt, 470,2009,344120,7421754,3,11,7,8,7.5yr2.5/1, cl++, 1vf-fsbk, A2, 3f-

$1 \mathrm{vf}, 25,0.75,72,1.2,2.1,0.105,1.9,0.1$,

$, 6.5,43.6,37.5,77.02,1.79,1.79,<0.01,0.11,1.29,0.16,17, \quad, \quad,-12.9,-$

$65.6,810,0.88,1.61,1.019,78.9,-12.9,-65.6,810,17.5,1, \quad, \quad, 7.5,-$

$16.6,33.5$, none,217,2.18,60, , , , 1.41, , , ,37,53,35.5,45.8,31,19,49, ,

'BB-450-C', SA '1508, le4-1, o'livine-poor basalt/sabie

basalt, $470,2009,344120,7421754,11,31,21,20,7.5 y r 3 / 2, \mathrm{cl}+, 2 \mathrm{~m}-\mathrm{cosbk}, \mathrm{Bw} 1,2 \mathrm{vf}-$

$\mathrm{f}, 15$-Oct, $0.865,260,1.5,2.5,0.094,2.4,0.1$,

$, 6.7,54.5,49.2,69.87,1.56,1.56,<0.01,0.1,4.04,0.2,16, \quad, \quad,-11.9,-$

$133.2,1270,0.88,1.39,3.168,78.5,-11.9,-133.2,1420,,, 1,, \quad, \quad$,

$,, 1.89,52.5,-12.9,-$

$147,1590,1.3,1,147,200,112.1,28,29,43,40.2,2,0,0,0,5,93,0,0$

BB-450-C, SA 1509, le4-1, olivine-poor basalt/sabie

basalt, 470,2009,344120,7421754,31,54,43,23,7.5yr3/2, cl+,2m-coabk, Bw2, 1vf-

$\mathrm{f}-\mathrm{co}, 15,0.85,293,1.5,2.9,0.115,2.7,0.2$,

, 7.2,57.7,53.4,68.19,1.25,1.25,<0.01,0.09,3.67,0.16,14, , ,-12.3,-

$209.9,2200,0.88,0.95,2.446,66.7,-12.3,-209.9,2320,, \quad,, \quad, \quad, \quad$,

$,, 1.5,58.5, \quad,, 1.01, \quad,, 150,350,143,27,24,49,, \quad, \quad$,

'BB-450-C', SA 1510, le4-1, olivine-poor basalt/sabie

basalt, 470,2009,344120,7421754,54,70,62, 16, 7.5yr3/3, cl+, 1-2f-msbk, Bw3, 1vf$\mathrm{f}-\mathrm{m}-\mathrm{co}, 45,0.55,132,1.5,2.2,0.131,2,0.2$,

$, 7.5,61,54.8,65.72,1.8,1.8,<0.01,0.07,2.38,0.15,25, \quad, \quad,-12.3,-$

$197.6,2030,0.86,0.91,1.033,43.5,-12.3,-197.6,2180$, ,

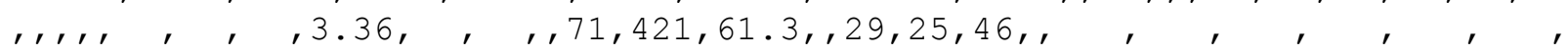

,

BB-450-C,SA 1511, le4-1, olivine-poor basalt/sabie

basalt, 470,2009,344120,7421754,70,85,78,15,7.5yr4/3, scl, M, 2Cr, 1vf, 45, 0.55 , $132,1.6,1.3,0.092,1.2,0.1$, $, 7.9,57.2,50.1,106.02,2.09,2.09,<0.01,0.05,2.76,0.18,14, \quad,-7.6,-$ $380,5070,0.8,0.59,0.625,22.6,-7.6,-380,5150$, , 
GR-550-C,SA 774,ST1,granite,550,2006,348678,7231971,0,15,8,15,7.5YR

3/2, sandy loam, 1 fm sbk, A, 2 vf m, 3,0.97,249,1.7,0.34,0.12,0.2, ,

, 5.1,5.2,2.7,36.63,0.7,0.72,<0.01,0.02,1.81,0.12,29, , ,-

$16.2,67.5,130,0.988,0.51,1.262,69.9,-18.1,50.3,170,16,1,, \quad, 16,-$

$22.2,57.7,1,152,,,,,,$,

$, 214,214,34.6,34.6,75,11,14,0,0,0,0,0,79,0,21,0$

GR-550-C,SA 775,ST1,granite,550,2006,348678,7231971,15,41,28,26,7.5YR

3/4, sandy loam, 1 fmco sbk,Bw1,1 co- 2 vffm, 5,0.95,448,1.8,0.36,0.12,0.2,

, $, 5.4,3.5,2.7,41.64,0.2,0.22,<0.01,0.01,1,0.04,19, \quad, \quad,-14.1,-$

$35.6,575,0.985,0.36,1.575,156.8,-17.1,59,148,14.45,,, \quad, \quad, 14.45,-$

$21.9,82.3,2.5,107,,, \quad, \quad, \quad, 385,600,62.2,96.7,72,14,14,, \quad$,

'GR-550-C,'SA 776,'ST1, granite, 550,2006,348678,7231971,41,62,52,21,7.5YR

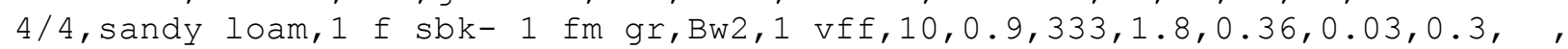

,5.6,4.7,4.2,37.01,0.2,0.16,<0.01,0.01,0.53,0.03,18, , ,-14.9,-

$85.6,1000,, 0.16,,,,-85.6,990,,,,,$,

$, \quad, 278,878,55.6,75,8,17,0.2,0,0,0,0,79,1,21,0$

GR-550-C,SA 777,ST1,granite,550,2006,348678,7231971,62,95,79,33,10YR

4/6, sandy loam, 1 vff gr- sg,2BC,1 vff,80,0.2,128,1.9,0.94,0.05,0.9, ,

, 5.6,5.7,5.2,25.47,0.2,0.15,<0.01,0.01,0.19,0.01,21, , ,-15.9,-

$170.6,1700,$, , , , , $-170.6,1850,,,,$, , , , , , , , ,

$\prime \prime \prime$
$\mathrm{GR}-550-\mathrm{C}, \mathrm{SA} 778, \mathrm{ST} 1, \mathrm{granite}, 550,2006,348678,7231971,95,105,100,10,10 \mathrm{YR}$

$5 / 4$, sandy loam/loamy sand, ma, 2C, $1 \mathrm{vf}, 80,0.2,37,1.9,1.54,0.24,1.3$, ,

,6.3,5.8,30.63,0.1,0.13,<0.01,0,0.05,0,28, , , $-16.4,-32.9,560, \quad, \quad$,

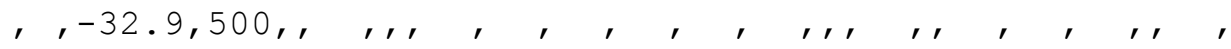

$, 37,1,81,8,11,, \quad, \quad, \quad$,

GR-550-S, SA 512, ST5, granite,550,2006,348755,7231990,0,2,1,2,10YR 6/2,10amy sand, $, A, \quad, 2,1,38,1.9,0.06,0.02,0$, ,

$, 6.2,4.9,2.6,64.37,0.7,0.66,<0.01,0.05,0.25,0.13,14, \quad, \quad,-$

$20.2,72.3,125,0.99,0.48,0.18,71.8,-18.8,62.9,140,22.49,,, \quad, \quad, 22.49,-$

$20.8,62.4,1,135,,, \quad, \quad, \quad, \quad$,

$, 36,36,2.1,2.1,82,13,6,0.9,0,0,0,0,76,17,7,0$

GR-550-S,SA 513, ST5, granite,550,2006,348755,7231990,2,10,6,8,10YR

6/3, sandy loam/loamy sand, ,Bw1, ,4,1,1512,1.9,0.06,0.04,0, ,

, 5.5,1.9,0.7,94.51,0.4,0.35,<0.01,0.04,5.36,0.07,10, , ,-

$19,63,140,0.99,0.4,6.004,112,-19.1,96.1,88,21.31,,, \quad, \quad, 21.31,-$

$20.2,82.1,2.5,107,,, \quad, \quad, \quad, \quad, 1386,1422,126,128.1,78,14,8,, \quad, \quad$,

'GR-550-S', SA '514, ST5, granite, 550,2006,348755,7231990,10,20,15, 10,10YR

$6 / 3$, loamy sand, ,Bw2, ,10,1,1890,1.9,0.06,0.02,0, ,

$, 4.9,3.5,2.7,36.16,0.3,0.25,<0.01,0.03,4.76,0.05,10, \quad, \quad,-17.7,53.6,160$,

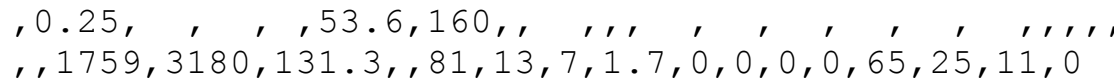

GR-550-S, SA 515, ST5, granite, 550,2006,348755,7231990,20,30,25,10,10YR

$6 / 3$, loamy sand, ,Bw3, ,3,1,1890,1.9,0.05,0.02,0, ,

$, 4.9,4.3,3.7,31.54,0.2,0.18,<0.01,0.03,3.42,0.03,7, \quad, \quad,-18.2,36,205$,

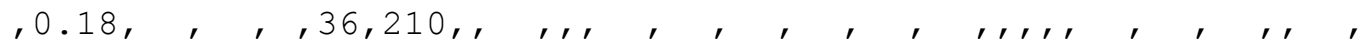

, 1733,4913,157.5, ,81,11,8, , ', ', ,

GR-550-T,SA 516, ST5, granite, 550,2006,348755,7231990,30,41,36,11,10YR

7/2, loamy sand, ,Bw4, ,4,1,2079,1.9,0.04,0.01,0, ,

$, 5,3.3,3,20.32,0.1,0.1,<0.01,0,1.98,0.02,48, \quad, \quad,-18.7,18.5,265, \quad, 0.1$,

, ', 18.5,270,', ', ', ', ', ', ', 
GR-550-T, SA 517, ST5, granite, 550,2006,348755,7231990,41,56,49,15,10YR $8 / 2$, loamy sand, ,BC1, ,23,1,2835,1.9,0.03,0,0, $, 5.5,5.1,4.8,13.23,0.1,0.08,<0.01,0,2.4,0.02,50$, $, \quad, \quad,-60.8,770$, $, 78,17,6$, GR-550-T, SA 518, ST5, granite, 550,2006,348755,7231990,56,70,63,14,10YR $8 / 2$, sand, ,BC2, ,93,0.9,2381,1.9,0.04,0,0, , , , , ,0.2,0.18,<0.01,0.07,4.31,0.03,3, , ,-19.7,-195.1,2000, , , , , $195.1,2100, r, r$ , , , , , , , GR-550-T,SA 740,ST10,granite, 550,2006,348831,7231986,0,8,4,8,7.5YR

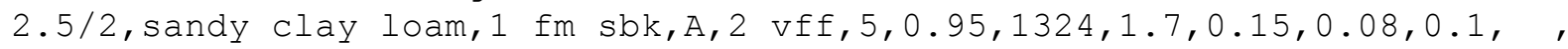
,6.5,10.1,7.4,65.9,0.8,0.8,<0.01,0.03,10.59,0.13,24, , ,$16.7,58,150,0.96,0.76,9.648,91.1,-16.7,58,150,21.16,,, \quad, \quad, 21.16,-$ $18,54.7,1,157,1,,,,$, $, 993,993,331,331,61,14,25,6.5,0,0,0,0,57,26,17,0$ GR-550-T,SA 741,ST10, granite,550,2006,348831,7231986,8,15,12,7,7.5YR

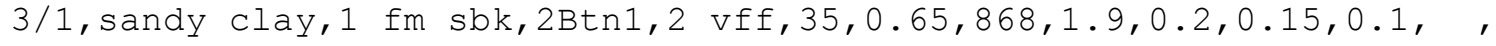
,6.5,21.4,19.1,56.84,0.7,0.7,<0.01,0.02,6.04,0.09,29, , ,$14.1,39.6,195,0.98,0.49,4.151,68.7,-17.4,79,110,15.17,1, \quad, \quad, 15.17,-$ $19.9,74.4,2,120,,,,,, \quad,, 530,1523,337.4,668.4,53,8,39,, \quad, \quad$

'GR-550-T', SA 742, ST10, granite, 550,2006,348831,7231986,15,29,22,14,10YR $3 / 2, \mathrm{clay}, 2 \mathrm{mco} a b k, 2 \mathrm{Btn} 2,2 \mathrm{vff}-1 \mathrm{vco}, 15,0.85,2296,1.9,0.16,0.07,0.1$, , $, 6.8,34.8,33.1,55.65,0.5,0.48,<0.01,0.02,11.12,0.08,24, \quad, \quad$, $13.8,2.6,340,, 0.3, \quad, \quad, 2.6,345$, , $, \quad, 1212,2735,1084,44,8,47,10.9,0,0,0,0,53,23,15,10$ GR-550-T, SA 743,ST10, granite,550,2006,348831,7231986,29,46,38,17,10YR 2/1, sandy clay loam, $1 \mathrm{~m}$ abk- ma,2Btn3,1 vff, 15,0.85,2730,1.9,0.27,0.04,0.2, , $, 7.6,42.5,41.5,60.35,0.3,0.3,<0.01,0.02,8.08,0.05,17, \quad, \quad,-13.8,1,345$, $, 0.09, \quad, \quad, 1,355$, , $, 1403,4138,1327.2,45,6,49$,

GR-550-T, SA 744, ST10,granite, 550,2006,348831,7231986,46,55,51,9,10YR $6 / 4$, sandy loam, $1 \mathrm{f}$ sbk- sg, 2Cr, $1 \mathrm{f}, 20,0.8,1436,2,0.33,0.01,0.3$, , 8.9,13.8,13.5,78.84,0.1,0.09,<0.01,0,1.25,0.01,31, , , $-13.8,-6,380, \quad$,

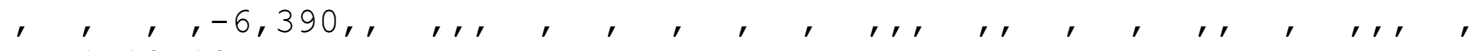
$, 71,10,19$, GA-740-C1, SA 1625, pkop3a-

3, gabbro, $740,2010,329124,7218015,0,4,2,4,10 y r 2 / 2$, sl, 1 fgr, A, , 5, 0.95,418,1.1,2.2,1.72,0.5,0.2,43,7.1,30.8,23.4,60.38,2.18,2.18,<0.01,0. $17,9.1,0.23,13, \quad,-22.3,-11.8,-62.1,755,0.97,1.58,6.405,70.4,-$ $13.4,78.2,112,0.006,34.72,0.943,10.4, \quad, \quad, \quad, 34.72,-$ $16.9,72.6,1,134,0.96,8.8,-17.6,-91.7,1040,2.48,-11.2,-$ $59.2,750,334,334,83.6,83.6,62.5,17.5,20,12,0,1,0,14,10,60,6,9$ GA-740-C1, SA 1626, pkop3a3, gabbro, 740,2010,329125,7218016,4,24,14,20,10yr2/2, scl, 1f-msbk, Bw1, $, 30,0.7,2100,1.5,2.7,2.12,0.6,0.3,42,7.1,45.1,38.4,63.59,1.97,1.97,<0.01,0$ $.16,41.33,0.21,12, \quad,-20.6,-13,-99.1,1100,0.95,1.51,30.146,72.9,-$ $11.8,6.1,325,0.009,23.24,4.499,10.9, \quad, \quad, \quad, 23.24,-$ $14.8,40.5$, none, 195, 0.87,11,-15.5,-129.9,1400,2.34,-12.7,$95.3,1050,1575,1909,525,608.6,60,15,25,17,0,1,0,8,16,68,6,1$ GA-740-C1, SA 1627, pkop3a3, gabbro, $740,2010,329126,7218017,24,44,34,20,10 y r 3 / 4, s 1,1-2$ fsbk, Bw2, 
$, 30,0.7,2100,1.5,2.5,2.45,0,0.5,33,7.4,43.1,39.9,63.42,0.95,0.95,<0.01,0.0$ $8,20.02,0.1,12, \quad, \quad-16.7,65.8,134,, 0.75, \quad, \quad,-15.3,-62.1,780$, , , , $, \quad, \quad, \quad, 1,2.14,22.4,-13.1,-171.3,1900,0.82,-$

$17.7,134.2,1890,3799,210,175,15,10,7.7,0,0,0,14,3,77,3,3$

GA-740-C1, SA 1628, pkop3a-

3, gabbro, 740,2010,329127,7218018,44,62,53,18,10yr3/4,sl,1fgr-sg-M, BC, $, 50,0.5,1350,1.5,1.8,2.06,-$

$0.3,0.3,28,7.6,32.1,30.4,65.9,0.49,0.49,<0.01,0.05,6.67,0.04,10, \quad, \quad,-$

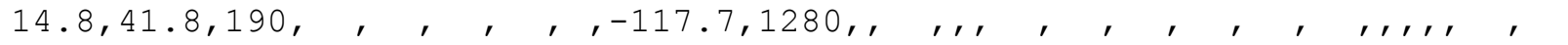
$,,,,,,,, 82.5,10,7.5,5.2,0,0,0,8,12,69,8,2$

GA-740-C1, SA 1629, pkop3a-

3, gabbro, 740,2010,329128,7218019,62,86,74,24, green, s, M-sg, Cr, $, 70,0.3,1152,1.6,1.2,1.78,-$

$0.6,0.3,42,7.9,18.1,17.4,83.35,0.2,0.2,<0.01,0.01,2.34,0.01,41, \quad, \quad,-$ $17.8,63.1,140,,,,,,-99.1,1120$,

, , , , , , ,87.5,7.5,5, , , , ,

GR-740-C,SA 119,ptkla, granite, $740,2004,326823,7211630,0,8,4,8,10 \mathrm{YR}$

$2 / 2,1 \mathrm{~s}, 0 \mathrm{sg} / 1 \mathrm{vfsbk}$,

,2vff, 0,1,1200,1.5,0.4,0,0.4,0,0.11,6.5,32.8,24.9,5,2.3,2.34,<0.01,0.16,28

$.08,0.35,15, \quad, \quad,-19.8,142.8,50,0.96,0.99,11.504,41,-$

$16.8,143.9,45,, 32.73,,, \quad, \quad, 32.73,-21.3,145.8,18,47,,,,,,$, , $, 1170,1170,30,30,80,17.5,2.5$, ,

GR-740-C,SA 120, ptkla, granite, $740,2004,326823,7211630,8,17,13,9,7.5 Y R$

$3 / 3, \mathrm{~s}, 1 \mathrm{vfmsbk} / 0 \mathrm{sg}$,

,3vff1c, 2,0.98,1499,1.7,0.4,0,0.4,0,0.01,6.1,8,6.4,18.27,0.5,0.48,<0.01,0. $04,7.13,0.08,12, \quad, \quad,-15.8,94.9,92,0.98,0.32,4.683,65.7,-$

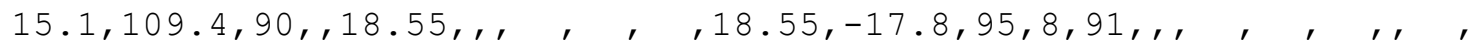
, ,1462,2632,37.5,67.5,82.5,15,2.5, , , , , , ', GR-740-C,SA 121,ptkla, granite, 740,2004,326823,7211630,17,39,28,22,7.5YR $4 / 4, \mathrm{~s}, 0 \mathrm{sg} / 1 \mathrm{mcsbk}, \quad, 2 \mathrm{vffmc}, 5,0.95,3219,1.5,0.5,0,0.4,0$, $, 5.9,13,12,3.14,0.3,0.31,<0.01,0.03,9.86,0.04,12, \quad, \quad,-16.8,78.9,115$, ,0.31, , ,-16.8,78.9,115,, , , , , , , , , , , , , $\prime, 3058,5690,160.9,, 77.5,17.5,5, \prime$
GR-740-C,SA 122,ptk1a, granite, $740,2004,326823,7211630,39,70,55,31,7.5 \mathrm{YR}$ GR-740-C,SA 122, ptkla, granite, 740,2004,326823,7211630,39,
$4 / 6$, s, 1mcsbk/0sg, , 3vffmc, 5, 0.95, 4741, 1.6, 0.4,0,0.4,0, $, 5.8,3.8,3.5,4.41,0.1,0.08,<0.01,0.01,3.69,0.01,11, \quad, \quad,-18.5,-$ $131.1,1260, \quad 0.08, \quad, \quad,-18.5,-131.1,1430$, , , , , , ,4623,10312,118.5, ,85,12.5,2.5, , , , , , GR-740-C,SA 123,ptkla,granite, 740,2004,326823,7211630,70,93,82,23,7.5YR $5 / 6$, s, 1mcsbk, ,3vfflmc, 10,0.9,3705,1.8,0.5,0,0.5,0, $, 5.2,1.7,1.5,56.73,0.1,0.07,<0.01,0.01,2.51,0.01,10, \quad, \quad,-18.4,-$ $172.6,1720$, , , , , $-18.4,-172.6,1900$, , , , , , , , , , , , , , 'GR-740-C,SA' 124, ptk1a, granite, $740,2004,326823,7211630,93,115,104,22,5 \mathrm{YR}$ $5 / 4, \mathrm{~s}, 1 \mathrm{mcsbk}, \quad, 2 \mathrm{vff} 1 \mathrm{mc}, 80,0.2,752,1.7$ $, 5.1,11.5,11.2,10.31,0.1,0.09,<0.01,0.01,0.71,0,10$, ', '76.25, '13.75,10,', ', ', ', ', ', ', GR-740-C,SA 125,ptkla, granite, 740,2004,326823,7211630,115,142, 129,27,5YR $5 / 4, \mathrm{~s}, 2 \mathrm{msbk}, \quad$, $\mathrm{fmlvc}, 65,0.35,1616,1.7, \quad, \quad$, , $, 5.4,8.6,8.4,14.47,0.1,0.06,<0.01,0.01,0.99,0,10$, ', $, 72.5,16.25,11.25, '$ 
GR-740-C,SA 126, ptkla, granite, 740,2004,326823,7211630,142,164, 153,22,5YR $4 / 4,1 \mathrm{~s}, 1 \mathrm{cmsbk}, \quad, 1 \mathrm{fm}, 65,0.35,1317,1.7$,

, 5.7,11.2,11,16.28,0.1,0.06,<0.01,0.01,0.76,0,8,

, , , , , , ,

$, 72.5,16.25,11.25$,

GR-740-C,SA 127,ptk1a,granite, 740,2004,326823,7211630,164,184, 174,20,5YR

$4 / 6, \mathrm{sl}, 2 \mathrm{cmsbk}, \quad, .5 \mathrm{fm}, 70,0.3,1062,1.8, \quad, \quad, \quad$,

, 5.9,11.1,10.8,23.83,0.1,0.06,<0.01,0.01,0.65,0,7, , , -20.1,-407.5,5700,

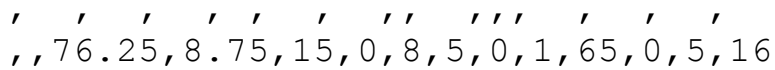

GR-740-C,SA 128,ptk1a, granite, 740,2004,326823,7211630,184,205,195,21,5YR

$4 / 6, \mathrm{sl}, 1 \mathrm{msbk} / 2 \mathrm{csbk}, \quad, .5 \mathrm{~m}, 65,0.35,1338,1.8$,

$, 5.7,13.8,13.6,20.55,0.1,0.05,<0.01,0.01,0.73,0,8$

, , , , , , , ,

$, 67.5,20,12.5$, ,

GR-740-C,SA 129,ptk1a,granite, 740,2004,326823,7211630,205,230,218,25,7.5YR $4 / 6, \mathrm{sc}, 1 \mathrm{mcsbk} / 0 \mathrm{sg}, \quad, .5 \mathrm{f}, 35,0.65,2909,1.8, \quad, \quad, \quad$, ,6.2,14.6,14.4,18.97,0,0.05,<0.01,0,1.4,0.01,10, , , $-21.9,-424.3,6085$,

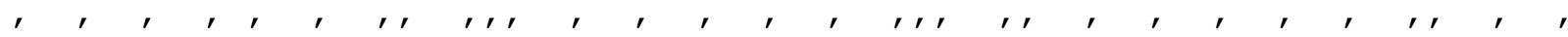
$,, 62.5,25,12.5$, GR-740-C,SA 130,ptk1a, granite, 740,2004,326823,7211630,230,245,238,15,7.5YR $5 / 6, \mathrm{~s}, 0 \mathrm{ma}, \quad, \quad 45,0.55,1419,1.7$, $, 6.2,14.4,14.3,18.86,0,0.03,<0.01,0,0.46,0,9$,

$,-23.5,-555.2,10190$,

', $, 75,17.5,7^{\prime} .5, '$,

MG-550-C1, SA 1630, sb7a-2, mixed

granite/gabbro,550,2010,341298,7232342,0,3,2,3,10yr3/3,sl, 1f-vfgr,A, 1-2vf$\mathrm{f}, 10,0.9,297,1.1,1.3,0.93,0.4,0.1,48.7,7,16.9,12.7$,

$, 1.25,1.25,<0.01,0.11,3.71,0.12,12, \quad, \quad,-13.5,-42,620,, 1.25, \quad$,

$, 65.8,135,,,$,

'MG-550-C'1, SA' $1631^{\prime}$, sb7a-2, mixed

granite/gabbro, 550,2010,341298,7232342,3,15,9,12,10yr3/3, sl, $1 \mathrm{f}-$

msbk, Bw1, 1f-vf-vc, 10,0.9,1728,1.6,1.3,1,0.3,0.2, 30, 7, 5.6,2.9,

$, 0.82,0.82,<0.01,0.07,14.1,0.12,12, \quad,-13.7,-77.4,910,, 0.82,$, ,

,41.8,190, , , , , , , , , , , , , , ,1469, 259.2, ,

$, 15,6.2,0,1,0,7,28,41,5,18$

$\mathrm{MG}-550-\mathrm{C} 1, \mathrm{SA} 1632, \mathrm{sb} 7 \mathrm{a}-2, \mathrm{mixed}$

granite/gabbro, 550,2010,341298,7232342,15,38,27,23, 7.5yr3/3,sl, $1 \mathrm{fgr-}$

$\mathrm{sg}, \mathrm{Bw} 2,1 \mathrm{f}-\mathrm{m}-\mathrm{co}-\mathrm{vc}, 70,0.3,1173,1.7,1.4,0.83,0.6,0.2,32,6.6,16.2,13.8$,

,0.71,0.71,<0.01,0.06,8.34,0.04,11, , ,-14.2,-65.5,810, ,0.71, , , , $65.5,790$,

'MG-550-C'1, SA' 1633, sb7a-2, mixed

granite/gabbro,550,2010,341298,7232342,38,60,49,22,2.5yr3/4, sl-scl, sg-

$\mathrm{M}, \mathrm{BC}, 1 \mathrm{vf}, 90,0.1,374,1.7,1.5,0.77,0.7,0.2,18,6.5,20.3,18.7$,

, 0.48,0.48,<0.01,0.05,1.78,0.01,9, , ,-13.9,-130.7,1250, , , , , , $130.7,1450$,

'MG-550-C2, SA' $1634^{\prime}, \mathrm{sb} 7 \mathrm{a}-3$, mixed

granite/gabbro,550,2010,341298,7232342,0,3,2,3,10yr3/3, sl, 1fgr, A, 1vf-

$\mathrm{f}, 5,0.95,314,1.1,1.2,0.88,0.3,0.1,71,6.7,13.6,9.9$,

$, 1.07,1.07,<0.01,0.09,3.37,0.11,12, \quad, \quad,-$

$17.8,63.1,140,0.99,0.91,2.829,84,-16.6,60.2,140,0.006,36.87,0.702,20.8, \quad$, 
$, \quad 36.87,-22.3,71.7,5.5,125,,,,,,,,, 266,266,47,$, ,

$, 15,7.2,1,1,0,6,26,48,2,15$

$\mathrm{MG}-550-\mathrm{C} 2, \mathrm{SA} 1635, \mathrm{sb} 7 \mathrm{a}-3, \mathrm{mixed}$

granite/gabbro,550,2010,341298,7232342,3,10,7,7,10yr2/2, sl-scl, 1fmsbk, Bw1,2vf-f, 10,0.9,1008,1.6,1.1,0.74,0.3,0.1,160,6.6, 16.4,13.7, $, 0.8,0.8,<0.01,0.07,8.03,0.11,11, \quad, \quad,-$

$16.8,0.2,360,0.98,0.75,7.393,92.1,-15.3,54.1,160,0.008,15.51,1.213,15.1$, $, \quad, 15.51,-20.6,80.5,6.8,110,1,15.7, \quad, \quad, \quad, 0.77,,, 882,1148,126$, , $, 12.5,6.6,1,1,0,5,21,53,2,17$

$\mathrm{MG}-550-\mathrm{C} 2, \mathrm{SA} 1636, \mathrm{sb} 7 \mathrm{a}-3, \mathrm{mixed}$

granite/gabbro,550,2010,341298,7232342,10,35,23,25,10yr3/4, scl,2msbk, BC1,2 $-3 \mathrm{vf}-\mathrm{f}-2 \mathrm{co}-\mathrm{vC}, 70,0.3,1275,1.7,1.3,0.89,0.5,0.2,60,7.2,20.7,18.1$, $0.75,0.75,<0.01,0.07,9.52,0.04,11, \quad, \quad,-13.5,-42,625, \quad, \quad, \quad$, $42,640,,, 1,,,,,, 1,0.71,9.6,-19.7,-322.4,4000,1,1,1,1$ $, \quad, 10,5.5,0,1,0,4,25,55,4,10$

$\mathrm{MG}-550-\mathrm{C} 2, \mathrm{SA} 1637, \mathrm{sb} 7 \mathrm{a}-3, \mathrm{mixed}$

granite/gabbro,550,2010,341298,7232342,35,60,48,25,7.5yr3/3,sl, sg-

$1 \mathrm{fsbk}, \mathrm{BC} 2,1 \mathrm{vf-f}, 90,0.1,425,1.7,1.2,0.52,0.7,0.2,31,7.2,17.6,15.5$, $, 0.62,0.62,<0.01,0.06,2.65,0.01,11, \quad, \quad,-13.7,-77.4,925, \quad, \quad, \quad$,

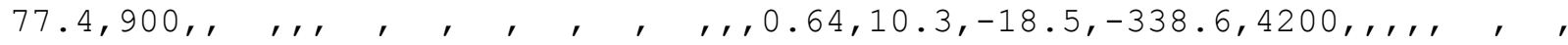
$, \quad, \quad, 10,6.3,0,1,0,4,26,63,1,5$

GA-550-C, SA 428, sb7a, gabbro,550,2005,333525,7230774,0,9,5,9,10YR 2/2, sl$\mathrm{sCl}, 2 \mathrm{f}-\mathrm{ms} b \mathrm{~b}-1 \mathrm{mgr}-1 \mathrm{vnpl}, \mathrm{BA}, 3 \mathrm{f}-\mathrm{vf}, 5,0.95,1197,1.4, \quad, \quad$, $, 0.11,7.15,36.7,30.4,41.83,1.9,1.86,<0.01,0.13,22.31,0.25,15, \quad, \quad,-$ $16.1,58.7,150,0.98,2.76,32.385,,-14.4,45.8,180,29.93,1,, \quad, 29.93,-$ $18.1,98.3,5,86,,,,,, 1,,,,, 220.9,22.5,12.5,1,$,

GA-550-C', SA 429, sb7a, gabbro, 550,2005,333525, 7230774,9,24,17, 15, $7.5 \mathrm{YR}$

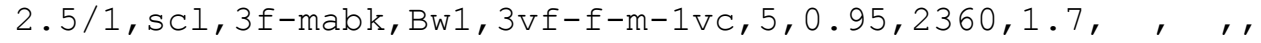
, 0.09, 7.44,42.5,37.2,49.54,1.5,1.54,<0.01,0.1,36.31,0.24,16, , ,$14.5,38.4,195,0.99,2.27,52.878, \quad, \quad 38.4,200,15.22,,, \quad, \quad, 15.22,-$ $13.2,88,4,98$,

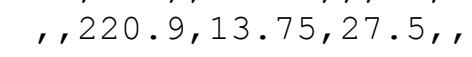

'GA-550-C', SA 430, sb7a, gabbro, 550,2005,333525, 7230774,24,43, 34, 19, $7.5 \mathrm{YR}$

$2.5 / 2, \mathrm{scl}, 3 \mathrm{f}-\mathrm{m}-\mathrm{co}-\mathrm{abk}, \mathrm{Bw} 2,1 \mathrm{vf}-\mathrm{m}, 5,0.95,2888,1.6, \quad, \quad$,

$, 0.14,7.37,43.3,39.3,51.09,1.2,1.17,<0.01,0.08,33.67,0.18,14$,

, 1.17,

$, 220.9,20,27.5$,

$\mathrm{GA}-550-\mathrm{C}, \mathrm{SA} 431$, sb7a, gabbro, 550,2005,333525,7230774,43,66,55,23,7.5YR

$2.5 / 2, \mathrm{scl}, 2 \mathrm{f}-\mathrm{mabk}, \mathrm{Bw} 3,1 \mathrm{vf}-\mathrm{f}, 15,0.85,2957,1.5, \quad, \quad, \quad$,

$, 7.4,47.2,43.7,50.25,1,1.03,<0.01,0.07,30.33,0.13,14, \quad, \quad,,,, 1.03$,

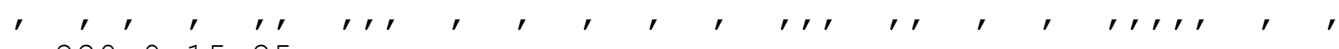

, 220.9,15,25, , , , , , , ,

$\mathrm{GA}-550-\mathrm{C}, \mathrm{SA} 432$, sb7a, gabbro, 550,2005,333525,7230774,66,84,75, $18,7.5 \mathrm{YR}$

$2.5 / 3, \mathrm{scl}, 2 \mathrm{vf}-\mathrm{fsbk}, \mathrm{Bw} 4,1 \mathrm{vf}, 25,0.75,2080,1.5, \quad, \quad$,

,0.1,7.51,119.8,117.6,19.86,0.6,0.65,<0.01,0.05,13.49,0.07,14, , ,

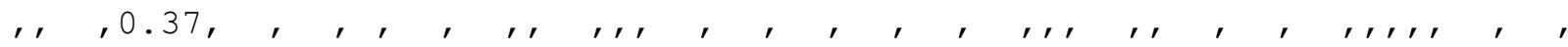
, 220.9,17.5,17.5, , , , , , , , , , , , , , , , , , , , , ,

GA-550-C, SA 433, sb7a, gabbro, 550,2005,333525,7230774,84,110,97,26,5YR

4/3,sl, ,"M, sg", ,57,0.43,1677,1.5, , , , , 0.08,7.73,44.4,43.2,54,0.4,0.37,<0.01,0.03,6.28,0.02,15, , , , ,

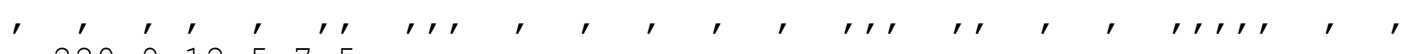

$, 220.9,12.5,7.5,, \quad, \quad, \quad$, 
GA-450-C, SA 1601, ph4a-

2, gabbro, 470,2010,322035,7449339,0,2,1,2,10yr3/2, scl, 1f-mgr-pl, A, 1vf-

$\mathrm{f}, 2,0.98,216,1.1,1.7,0.14,1.6,0.1,94.9,7.9,26.8,21.3,100,1.62,1.62,0.01,3$ $.5,0.18,15, \quad, \quad,-16.6,22,250,0.96,2.75,5.708, \quad,-19.2,-$

$16.1,450,0.006,41.08,0.537,15.3, \quad, \quad, \quad, 41.08,-$

$19.2,61,3.5,145,1.29,11.9,-16.3,-4.4,380,1.68,-$

$16.7,25.6,240,183,183,32.3,220.9,30,15,1.8,36,8,0,26,0,12,0,18$

GA-450-C, SA 1602 , ph4a-

2, gabbro, $470,2010,322035,7449339,2,11,7,9,10 y r 3 / 2$, scl, 2f-msbk, Bw1,2-3vf-f-

$1 \mathrm{~m}, 5,0.95,1197,1.4,1.9,0.19,1.7,0.2,126.2,8.1,32.2,25.8,82.9,1.86,1.86,0.0$

$1,22.29,0.25,19, \quad, \quad,-14.5,-0.1,350,0.95,1.07,12.174,54.6,-17,-$

$0.1,360,0.009,35.11,3.656,16.4, \quad, \quad, \quad, 35.11,-17,54.4,2,160,1,7.4, \quad, \quad$,

$, 2,1,1032,1216,164.6,220.9,24,14,5.6,9,3,0,9,3,41,6,29$

GA-450-C, SA $1603, \mathrm{ph} 4 \mathrm{a}-$

2, gabbro,470,2010,322035,7449339,11,20,16,9,10yr2/2,scl, 1f-vfsbk-

$1 \mathrm{mgr}, \mathrm{Bw} 2,2 \mathrm{vf}-\mathrm{f}-1 \mathrm{~m}-$

$\mathrm{CO}, 10,0.9,1215,1.5,2.2,0.18,2,0.2,117.3,8.3,36.1,29.1,130.92,2.06,1.74,0.3$ $2,21.09,0.23,20, \quad,-14.3,-54.6,710,, 1.74, \quad, \quad,-54.6,710,,, 1$,

$, \quad, \quad, 1,1,2.9, \quad$,

, 1.77, , , 1154,2370,60.8, 220.9,38,5,3.1,2,1,0,9,8,62, 2, 16

GA-450-C, SA 1604, ph $4 a-$

2, gabbro, 470,2010,322035,7449339,20,34,27,14,10yr4/2, scl, 1m-cogr, Bw3, 1vf$\mathrm{f}-$

$\mathrm{m}, 35,0.65,1365,1.5,1.7,0.15,1.6,0.2,112,8.4,32.9,23.2,153.56,2.84,1.43,1.4$ $1,19.57,0.14,20, \quad,-15.9,-70.8,825,, 1.43, \quad, \quad,-70.8,850,,,,$, , $,,,, 1,0.8,5.6,,$,

$, 1.5,1,1229,3598,136.5,220.9,40,10,6.4,1,1,26,4,3,64,0,2$

GA-450-C, SA 1605, ph 4 a-

2, gabbro, 470,2010,322035, 7449339,34,48,41,14,10yr4/4, sl-scl 20\%, 1vfgr-

$\mathrm{sg}, \mathrm{BC} 1,1 \mathrm{vf-f-}$

$\mathrm{m}, 30,0.7,1568,1.6,1.2,0.1,1.1,0.2,106,8.5,24.9,16.3,213.24,2.53,0.73,1.8$, ,

$11.47,0.08,16, \quad,-15.9,-103,1125$,

, , , , , , , , , , , , , , , , , , $20.9,20,15,9.3,0,2,29,2,3,62,0,1$

GA-450-C, SA 1606, ph 4 a-

2, gabbro, 470,2010,322035, $7449339,48,70,59,22,10 \mathrm{yr} 4 / 6, \mathrm{sl}<15 \%, \mathrm{sg}, \mathrm{BC} 2,1 \mathrm{vf}, 45$, $0.55,1936,1.6,1,0.09,0.9,0.1,103,8.6,22.5,15,216.73,2.19,0.4,1.79,0.04,7.7$ $2,0.04,10, \quad,-15.1,-138.8,1330,, 1.7, \quad, \quad,-138.8,1500$, ,

, , , , , , , , , , , , , , , , , , , , , , , , , ,

, ,

$\mathrm{GA}-740-\mathrm{C}, \mathrm{SA} 1620, \mathrm{pkop} 3 \mathrm{a}-$

2, gabbro, $740,2010,329124,7218015,0,3,2,3,10 y r 3 / 2$, sl-scl, 1 fsbk-1fgr, A, 36vf$\mathrm{f}, 5,0.95,314,1.1,2.2,0.77,1.5,0.2,35,6.9,19.5,13.5$,

$, 1.76,1.76,<0.01,0.14,5.53,0.18,13, \quad,-20.8,-$

$13.6,88.1,98,0.97,1.55,4.697,85,-13.1,85.2,105,0.007,35.65,0.78,14.1,$,

$, \quad, 35.65,-16.1,67.6,4,133,1.17,14.8,-19.1,-160,1720,1.93,-$

$12.7,131.1,53,244,244,69.9,, \quad, \quad, 27.5,19.8,0,1,0,15,6,72,3,4$

GA-740-C, SA 1621, pkop3a-

2, gabbro, $740,2010,329125,7218016,3,9,6,6,10 y r 3 / 3$, sl-scl, 1 fsbk, Bw1, 2vf-f$\mathrm{VC}, 10,0.9,648,1.2,2.7,2.12,0.6,0.3,55,7.1,32.2,24.6$,

,2.24,2.24,<0.01,0.18,14.48,0.24,12, ,-16.1,-

$13.4,70,123,0.96,1.77,11.018,76.1,-12.8,60.6,145,0.01,35.27,2.307,15.9, \quad$, $, \quad, 35.27,-16.9,64.4,3.5,137,1.1,10.3,-17.7,-116.1,1270,2.54,-$

$12.9,91.4,96,513,756,135.5,, \quad, \quad, 27.5,19.8,0,0,0,14,8,72,3,3$ 
GA-740-C, SA 1622, pkop3a-

2, gabbro, 740,2010,329126,7218017,9,25,17,16,10yr3/4, scl, 1f-msbk, Bw2, 1vf$\mathrm{f}, 15,0.85,2040,1.5,2.4,2.18,0.2,0.3,44,7.4,40.5,36.4$,

$, 1.21,1.21,<0.01,0.1,24.61,0.15,12, \quad,-16.9,-13.2,-12.1,425, \quad, 1.21, \quad, \quad$ $,-12.1,430,,,,, \quad, \quad, \quad, 78.5,6,113,1,13.9, \quad$, ,

$1.25,, 1,1698,2454,342.4,, \quad, 25,, \quad, \quad, \quad$, , 
Table S2. Correlation matrices (using Hmisc package in R; Harrell et al. 2016). Number of observations $=15$; Table 5 was the basis for the correlation matrix. Geology was assigned numeric values from felsic to mafic lithologies $(1=$ rhyolite, $2=$ granite, $2.5=$ mixed granite, $3=$ gabbro, $4=$ nephelenite, $5=$ basalt). Depth $=$ maximum soil depth used in the study. CEC $=$ cation exchange capacity corrected for organic $\mathrm{C}$ contribution (see text). Clay $=$ mass fraction of soil that is in the clay-sized fraction; Smec $=\%$ of clay that is smectite; clay.smec $=$ Clay* smectite (i.e. the average amount (in \%) of smectite clay in the profile). Fed and Feo are the citratedithionite and oxalate extractable $\mathrm{Fe}$ fractions expressed as per cent. (In general extractable $\mathrm{Al}$ was much lower and not considered). $13 \mathrm{C}$ is the profile-averaged $\delta^{13} \mathrm{C}(\%)$ and $14 \mathrm{C}$ the profile-averaged $\Delta^{14} \mathrm{C}(\%)$. TT is estimated turnover time (in years) and was derived from the $\mathrm{C}$-weighed averages as were the isotopic values.

Significance is indicated as $* \mathrm{p}<.05, * * \mathrm{p}<.01, * * * \mathrm{p}<.001$

\begin{tabular}{|c|c|c|c|c|c|c|c|c|c|c|c|c|c|c|c|}
\hline & geology & rainfall & $\mathrm{Fe}(\mathrm{d})$ & $\mathrm{Fe}(\mathrm{o})$ & $\begin{array}{l}\text { Fe.d - } \\
\text { Fe.o. }\end{array}$ & $\mathrm{pH}$ & $\begin{array}{c}\text { Cless_ } \\
\text { CEC }\end{array}$ & $\begin{array}{l}\text { ORGA } \\
\text { NIC.C }\end{array}$ & $\mathrm{mC} . \mathrm{N}$ & $\begin{array}{c}\text { mean } \\
13 C\end{array}$ & $\begin{array}{l}\text { mean } \\
\text { D14C }\end{array}$ & $\begin{array}{c}\text { TT } \\
\text { (year) }\end{array}$ & $\begin{array}{l}\text { Fclay- } \\
\text { sized }\end{array}$ & $\begin{array}{c}\text { mean } \\
\text { Clay } \\
\text { content }\end{array}$ & $\begin{array}{c}\text { phosph } \\
\text { ate }\end{array}$ \\
\hline \multicolumn{16}{|l|}{ geology } \\
\hline rainfall & -0.34 & & & & & & & & & & & & & & \\
\hline $\mathrm{Fe}(\mathrm{d})$ & $0.57^{*}$ & -0.27 & & & & & & & & & & & & & \\
\hline $\mathrm{Fe}(\mathrm{o})$ & 0.28 & 0.44 & 0.28 & & & & & & & & & & & & \\
\hline Fe.d - Fe.o. & 0.53 & -0.38 & $0.97 * * *$ & 0.06 & & & & & & & & & & & \\
\hline $\mathrm{pH}$ & $0.62^{*}$ & -0.25 & 0.46 & 0.28 & 0.42 & & & & & & & & & & \\
\hline Cless_CEC & $0.77^{* *}$ & -0.19 & $0.72^{* *}$ & 0.21 & $\begin{array}{c}0.70 * * \\
0.88\end{array}$ & $0.59 *$ & & & & & & & & & \\
\hline ORGANIC.C & $0.7^{* *}$ & -0.28 & $0.91 * * *$ & 0.26 & $* * *$ & 0.53 & 0.84 & & & & & & & & \\
\hline $\mathrm{mC} . \mathrm{N}$ & -0.07 & -0.53 & -0.2 & -0.43 & -0.11 & -0.14 & -0.01 & -0.07 & & & & & & & \\
\hline mean $13 \mathrm{C}$ & $\begin{array}{l}0.52 \\
-0.83\end{array}$ & -0.21 & 0.32 & 0.16 & 0.29 & $0.78 * *$ & 0.47 & 0.37 & 0.2 & & & & & & \\
\hline mean $\mathrm{D} 14 \mathrm{C}$ & $* * *$ & 0.35 & -0.29 & -0.17 & -0.26 & $-0.6 *$ & -0.55 & -0.33 & -0.07 & $-0.7^{* *}$ & & & & & \\
\hline TT (year) & $0.79 * *$ & -0.19 & 0.36 & 0.3 & 0.3 & $0.83^{* * *}$ & $0.61 *$ & 0.45 & -0.12 & $0.66^{*}$ & $-0.82 * * *$ & & & & \\
\hline $\begin{array}{l}\text { Fclay-sized } \\
\text { mean Clay }\end{array}$ & 0.16 & 0.14 & -0.32 & -0.32 & -0.26 & -0.05 & 0.23 & -0.2 & 0.16 & 0.09 & -0.32 & 0.27 & & & \\
\hline $\begin{array}{l}\text { content } \\
\text { smectitie }\end{array}$ & $0.64 *$ & -0.29 & 0.35 & -0.02 & 0.37 & 0.4 & $0.83 * * *$ & 0.49 & 0.16 & 0.41 & $-0.61 *$ & $\begin{array}{c}0.5 \\
0.84^{* *}\end{array}$ & 0.53 & & \\
\hline (fraction of & $0.92 * * *$ & -0.24 & 0.39 & 0.3 & 0.34 & $0.64 *$ & $0.73^{* *}$ & 0.53 & -0.07 & 0.47 & $-0.85 * * *$ & $*$ & 0.24 & $0.62 *$ & \\
\hline
\end{tabular}


clay)

phosphate

content

$0.79 * * \quad-0.47$

0.38

0.15

0.36

0.42

0.54

0.48

0.03

0.18

$-0.54$

$0.62 * \quad 0.19$

0.53

$0.67^{*}$

Clay*smec

$0.85 * * * \quad-0.32$

$\begin{array}{ll}0.39 & 0.02\end{array}$

0.48

$\begin{array}{lll}0.79 * * & 0.48 & 0.07\end{array}$

0.44

$\begin{array}{lll}-0.83 * * * & 0.73 * * & 0.54\end{array}$

$0.84 * * *$

$0.87^{* *}$ 


\section{Code for generating radiocarbon for a given TT.}

For additional information on SoilR please see Sierra et al. 2014 (in main text references).

S O I L R version 1.1 can be downloaded from the Comprehensive R Archive Network (CRAN) or RForge. Source code and test framework can be obtained from these two repositories. To install, use the function install.packages("SoilR",repo), specifying either a CRAN mirror or RForge in the repo argument.

\#\#\#\# R Code for determining the 14C of a steady state homogeneous, one-pool model \#\#\#\#\# uses the SoilR package and the Hua et al. (2013) Curve for the Southern Hemisphere \#\#\# First, install the SoilR package.

install.packages(“SoilR”, repo) \#\# repo is the repository (CRAN mirror or RForge, as needed)

\#\#\#\#\# Load the SoilR library

$\operatorname{library}(\operatorname{SoilR})$

\#Bind the IntCal13 dataset and Hua2013 for the Southern Hemisphere Zones 1,2 \# This produces the atmospheric 14C record from 50,000 BP to 2010 in Years AD

ad=bind.C14curves(prebomb=IntCal13,postbomb=Hua2013\$SHZone12,time.scale="AD")

\#\# Plot the atmospheric record

plot(ad[,1:2],type="1")

plot(ad[,1:2],type="1",xlim=c(0,2010))

abline $(v=1950,1$ ty $=2)$

\#\#\#\#\#\#\# To estimate the Value of 14C as a function of time for a given Turnover time (TT) \#\# Example given is for 50 year TT (you can change the value as needed) $\mathrm{TT}=50$ \#\#\# Put in the value of the TT in years you wish to use (in years)

\#\#\# Other factors will be calculated to make sure model is at steady state $\mathrm{k} 1=1 / \mathrm{TT}$ \#\#\# $\mathrm{k} 1$ is the decomposition rate $(1 / \mathrm{TT})$ in $1 / \mathrm{yr}$ la $=1 / 8267$ \#\#\# la is the radio-decay constant for radiocarbon $1 /$ mean life $\mathrm{Fz}=\mathrm{k} 1 /(\mathrm{k} 1+\mathrm{la}) \quad$ \#\#\# Steady state pre-bomb estimate of the Absolute Fraction Modern (see Sierra et al. 2014)

$\mathrm{DFz}=1000 *(\mathrm{Fz}-1) \quad$ \#\#\# Expressed as Delta $14 \mathrm{C}$

\#\#\#\#\# Other model inputs are calculated so as to have the model remain at steady state LitterInput $=10$ \# arbitrary inputs

Cinit=LitterInput*TT \# Inventory at steady state = initial Cinventory (arbitrary units) \#\#\#\# Next step is to run the model \#\# In SoilR the one pool model is a function that can be called years $=\operatorname{seq}(1901,2010$,by=0.5) \# time scale for running the model (expressed in years AD) 
$\mathrm{Ex}=$ OnepModel14(t=years, $\mathrm{k}=\mathrm{k} 1, \mathrm{C} 0=\mathrm{Cinit}, \mathrm{F} 0=\mathrm{DFz}, \mathrm{In}=$ LitterInput, inputFc $=\mathrm{ad}) \quad$ \#Soil $\mathrm{R}$ model function

C14t=getF14(Ex) \# Extracts $14 \mathrm{C}$ for each year

$\mathrm{Ct}=\mathrm{getC}(\mathrm{Ex}) \quad$ \# Extracts $\mathrm{C}$ inventory for each year (check for steady state)

$\mathrm{DEL}=\mathrm{C} 14 \mathrm{t}[217$,] \# This extracts the 14C signature in the year 2010

\#\# Next steps make a plot of $14 \mathrm{C}$ versus year

plot(C14Atm_NH,type="l",xlab="Year",ylab="Delta 14C (per mil)",xlim=c(1940,2010))

lines(years, $\mathrm{C} 14 \mathrm{t}[, 1]$, col $=4$ )

points(2010, DEL, cex=1.5)

legend(

"topright",

c("Delta 14C Atmosphere", "Delta 14C in SOM"),

lty $=\mathrm{c}(1,1)$,

$\mathrm{col}=\mathrm{c}(1,4)$,

$\operatorname{lwd}=\mathrm{c}(1,1)$,

bty="n"

)

\section{\#\#\#\#\#}

C14t[217,] \#This line will return only the Del14C for the year 2010 (to be compared with the measured value)

\#\#\#\#\#\#\#\#

\#\#\#\#\#\#\#\#

\#\#\# This code generates a table and a plot of the $14 \mathrm{C}$ signature

\#\#\#\# expected in 2010 for the one-pool, homogeneous, steady state model

\#\#\# assimung a range of TTs (1 to 2000 years).

\#\# This generates a "lookup" table for comparing with the data

soll=2000 \#\#\# This is the end TT, starts with 1 year

sols= data.frame $(1:$ sol1, 1:sol1 $) \# \#$ makes a data frame of the right size

for(i in 1:sol1) \#\# number of calculations

\{

$\mathrm{TT}=\mathrm{i}$

$\mathrm{k} 1=1 / \mathrm{TT}$

la $=1 / 8267$

$\mathrm{Fz}=\mathrm{k} 1 /(\mathrm{k} 1+\mathrm{la})$

$\mathrm{DFz}=1000 *(\mathrm{Fz}-1)$

LitterInput $=10$

Cinit=LitterInput*TT

Cinit

years $=\operatorname{seq}(1901,2010$, by $=0.5)$

$\mathrm{Ex}=$ OnepModel14$(\mathrm{t}=\mathrm{years}, \mathrm{k}=\mathrm{k} 1, \mathrm{C} 0=\mathrm{Cinit}, \mathrm{F} 0=\mathrm{DFz}, \mathrm{In}=$ LitterInput, inputFc $=\mathrm{ad})$

$\mathrm{C} 14 \mathrm{t}=$ getF14(Ex)

$\mathrm{DEL}=\mathrm{C} 14 \mathrm{t}[206$,

$\operatorname{sols}[\mathrm{i}, 1]=\mathrm{i}$ 
sols $[i, 2]=$ DEL

$\mathrm{i}=\mathrm{i}+1$

\}

\#\# Write the whole file

write.csv(sols, file $=$ "Solutions.csv")

\#\# make a plot of the 14C expected in 2010 for each TT.

plot(sols[,1],sols[,2],xlab="TT",ylab="Delta 14C (per mil)"

\section{References:}

Harrell, F.E., Jr, with contributions from Charles Dupont and many others.: Hmisc: Harrell Miscellaneous. R package version 3.17-2. https://CRAN.R-project.org/package=Hmisc, 2016.

Hua, Q., Barbetti, M., and Rakowski, A.: Atmospheric radiocarbon for the period 1950-2010, Radiocarbon, 55, 2059-2072, 2013.

Sierra, C., Müller, M.M., Trumbore, S.E.: Modeling radiocarbon dynamics in soils: SoilR version 1.1, Geoscientific Model Development 7 (5), 1919-1931, 2014. 